\title{
Energy Demodulation Algorithm for Flow Velocity Measurement of Oil-Gas-Water Three-Phase Flow
}

\author{
Yingwei Li, ${ }^{1}$ Jing Gao, ${ }^{1}$ Xingbin Liu, ${ }^{2}$ and Ronghua Xie ${ }^{3}$ \\ ${ }^{1}$ School of Information Science and Engineering, Yanshan University, Qinhuangdao 066004, China \\ ${ }^{2}$ Logging and Testing Services Company, Daqing Oilfield Limited Company, Daqing 163453, China \\ ${ }^{3}$ Daqing Oilfield Limited Company, Daqing 163412, China
}

Correspondence should be addressed to Yingwei Li; lyw@ysu.edu.cn

Received 17 January 2014; Revised 30 March 2014; Accepted 8 April 2014; Published 29 April 2014

Academic Editor: Huaiqin Wu

Copyright (C) 2014 Yingwei Li et al. This is an open access article distributed under the Creative Commons Attribution License, which permits unrestricted use, distribution, and reproduction in any medium, provided the original work is properly cited.

Flow velocity measurement was an important research of oil-gas-water three-phase flow parameter measurements. In order to satisfy the increasing demands for flow detection technology, the paper presented a gas-liquid phase flow velocity measurement method which was based on energy demodulation algorithm combing with time delay estimation technology. First, a gasliquid phase separation method of oil-gas-water three-phase flow based on energy demodulation algorithm and blind signal separation technology was proposed. The separation of oil-gas-water three-phase signals which were sampled by conductance sensor performed well, so the gas-phase signal and the liquid-phase signal were obtained. Second, we used the time delay estimation technology to get the delay time of gas-phase signals and liquid-phase signals, respectively, and the gas-phase velocity and the liquidphase velocity were derived. At last, the experiment was performed at oil-gas-water three-phase flow loop, and the results indicated that the measurement errors met the need of velocity measurement. So it provided a feasible method for gas-liquid phase velocity measurement of the oil-gas-water three-phase flow.

\section{Introduction}

Multiphase flow system is very common in nature and industrial processes, such as petroleum, metallurgy, chemical industry, energy resources, and biomedicine [1]. The oil-gaswater three-phase flow, as a multiphase flow of a fluid form, is prevalent in the oil and gas industry. The present research of multiphase flow field, especially the research on measurement technology of oil-gas-water three-phase flow at home and abroad, has become one of the most important topics in the researches of multiphase flow $[2,3]$. For the three-phase flow of industrial production system, most parameters of oilgas-water three-phase flow are with unpredictable characteristics. The accurate measurement of the flow rate affects the automatic control system, measurement accounting, and material and energy balance. They are all very important to the economic operation and the reliability of the engineering equipment.

Liu et al. [4] proposed a novel method for particle velocity measurement by using the correlation algorithm. In the method the predicted transit time was used to implement an adaptive process for selecting signal segments which were of higher overlap degree. The proposed algorithm improved the correlation coefficient and provided a way to detect the transit time with a higher accuracy. Simulations were carried out to evaluate the proposed adaptive algorithm by comparing it with the conventional algorithm. And the algorithm demonstrated that the adaptive correlation algorithm is potentially powerful for velocity measurement. Tan and Dong [5] raised a method in which a dual-plane electrical resistance tomography (ERT) was adopted to crosscorrelate the sensing data from two electrode planes. It was a method of dynamically seeking suitable signal segment for cross-correlating measured signals from two-phase flow. It proved that the cross-correlation velocity was a structural velocity within two-phase flow, and the relationship of crosscorrelation velocity and mixture velocity was affected by the water flow rate within the two-phase flow. Kong et al. [6] put forward a kind of oil-gas-water three-phase flow velocity measurement method based on ICA and time delay 
estimation. Due to the limitations of traditional ICA algorithm, using this method needed to collect four measurement signals for signal separation, which increased the system measurement complexity. Kan et al. [7] used the Monte Carlo method to build a three-dimensional dynamic model. Based on the dynamic model, the superposition of nonuniform electric field, and the normalized cross-correlation algorithm, the velocity of the dispersed phase was calculated out. Furthermore, the influence of the model parameters on the dispersed phase's velocity was measured. Related simulation experiments were conducted to validate the conclusion of the paper. Seraj et al. [8] used electrostatic sensors and the cross-correlation method for deriving the velocity of solid/air particles. Electrostatic sensors were providing a nonintrusive method for measuring the velocity of fluid in solid/gas applications and, therefore, were less susceptible to wear and erosion.

The correlation method was an effective way to measure velocity, since it was sensitive to the peak voltage in the sensor output and, therefore, was less sensitive to the noise. The correlation method could be realized by using different types of sensing element. Among them, electrostatic sensors were most widely used, due to their good performance and their nonintrusive arrangement. For oil-water two-phase flow or gas-water two-phase flow parameter detection, correlation measurement technique provided a technical method, but in oil exploration it was mostly the oil-gas-water three-phase flow; it was difficult to achieve the ideal results by only using correlation measurement technique [9-12]. In order to improve the oil-gas-water three-phase flow velocity measurement accuracy, combining blind signal separation technology with the correlation measurement technique for multiphase flow velocity measurement could be a solution to the problem. Because of the limitation of the complexity of the system, cost saving, and the number of receiving equipment, using only one sensor to collect signal was very common, thus the issue of separating original signal from the mixed signals which were received from a conductance sensor belongs to single-channel blind signal separation problem. To solve the problems in the processing of oil-gas-water threephase flow parameter measurement, we proposed a method which combines single-channel blind signal separation with correlation measurement technique for oil-gas-water threephase flow gas-phase and liquid-phase velocity measurement. By using energy demodulation algorithm $[13,14]$ on the oil-gas-water three-phase flow signal separation, we got the gas-phase signal and liquid-phase signal and then combined them with correlation measurement technology separately to calculate the time delays in gas-phase signal and liquid-phase signal. Finally we figured out the oil-gas-water three-phase flow gas-phase velocity and liquid-phase velocity.

\section{The Energy Demodulation Algorithm for Blind Signal}

2.1. Energy Operator Demodulation Algorithm. U.S. scientist Teager proposed a simple signal analysis algorithm on nonlinear speech modeling study, and this new algorithm was called energy operator [15]. Energy operator was very helpful in signal analysis either in a discrete domain or a continuous domain. The signal analysis method could simplify the calculation greatly and make expressions clearer.

The continuous signal energy operator for $x(t)$ is defined as

$$
\Psi_{c}[x(t)]=[\dot{x}(t)]^{2}-x(t) \ddot{x}(t) .
$$

In the formula $\dot{x}(t)$ is the first-order derivative of $x(t)$ and $\ddot{x}(t)$ is the second-order derivative of $x(t)$ about time.

For the general form of a continuous signal $x(t)=$ $a(t) \cos [\phi(t)], a(t)$ is the instantaneous amplitude of $x(t), \phi(t)$ is the instantaneous phase position of $x(t)$, and the derivative of $\phi(t)$ is the instantaneous frequency $w(t)$ of $x(t)$. Through the derivatives for $x(t)$, we can obtain

$$
\dot{x}(t)=\dot{a}(t) \cos \phi(t)-a(t) \dot{\phi}(t) \sin \phi(t) .
$$

Differentiate $\dot{x}(t)$ again, we have

$$
\begin{aligned}
\ddot{x}(t)= & \ddot{a}(t) \cos \phi(t)-2 \dot{a}(t) \sin \phi(t) \dot{\phi}(t) \\
& -a(t) \cos \phi(t)[\phi(t)]^{2}-a(t) \sin \phi(t) \ddot{\phi}(t) .
\end{aligned}
$$

Take $\dot{x}(t)$ and $\ddot{x}(t)$ into (1):

$$
\begin{aligned}
\Psi_{c}[x(t)]= & {[a(t) \phi(t)]^{2}+\frac{1}{2} a(t) \ddot{\phi}(t) \sin [2 \phi(t)] } \\
& +\cos ^{2} \phi(t)\left\{[\dot{a}(t)]^{2}-a(t) \ddot{a}(t)\right\} .
\end{aligned}
$$

If the signal magnitude and the change rate for frequency with time do not change too fast, or comparing with the carrier frequency the signal frequency is not very large, and $\dot{a}(t)=\ddot{a}(t)=\ddot{\phi}(t)=0$, the energy operator of $x(t)$ can be expressed as

$$
\Psi_{c}[x(t)] \approx[a(t) \phi(t)]^{2}=a^{2}(t) w^{2}(t) .
$$

Similarly, we can get

$$
\Psi_{c}[\dot{x}(t)] \approx a^{2}(t) w^{4}(t) .
$$

Combine formula (5) with (6) we can obtain

$$
\begin{aligned}
& w(t) \approx \sqrt{\frac{\Psi_{c}[\dot{x}(t)]}{\Psi_{c}[x(t)]}}, \\
& |a(t)| \approx \frac{\Psi_{c}[x(t)]}{\sqrt{\Psi_{c}[\dot{x}(t)]}} .
\end{aligned}
$$

The general form of mixed signal that contains two signal components can be expressed as

$$
x(t)=a_{1}(t) \cos \left(\int_{0}^{t} w_{1}(\tau) d \tau\right)+a_{2}(t) \cos \left(\int_{0}^{t} w_{2}(\tau) d \tau\right) .
$$

Mixed signal at a certain instantaneous time can be replaced by the signal containing two sinusoidal signal components [16], and the mixed signal can be expressed as

$$
x(t)=a_{1} \cos \left(w_{1} t+\theta_{1}\right)+a_{2} \cos \left(w_{2} t+\theta_{2}\right) .
$$


According to the sinusoidal signal differential relations, mixed signal $x(t)$ satisfies the following differential equations:

$$
x^{(4)}+c_{1} \ddot{x}+c_{2} x=0 .
$$

With $x^{(n)}=d^{n} x / d t^{n}$, tidy

$$
\begin{gathered}
\left(a_{1} w_{1}^{4}-c_{1} a_{1} w_{1}^{2}+c_{2} a_{1}\right) \cos \left(w_{1} t+\theta_{1}\right) \\
+\left(a_{2} w_{2}^{4}-c_{1} a_{2} w_{2}^{2}+c_{2} a_{2}\right) \cos \left(w_{2} t+\theta_{2}\right)=0, \\
a_{1} w_{1}^{4}-c_{1} a_{1} w_{1}^{2}+c_{2} a_{1}=0, \\
a_{2} w_{2}^{4}-c_{1} a_{2} w_{2}^{2}+c_{2} a_{2}=0 .
\end{gathered}
$$

Combine (12) with (13), we can obtain

$$
c_{1}=\left(w_{1}^{2}+w_{2}^{2}\right), \quad c_{2}=w_{1}^{2} w_{2}^{2} .
$$

Extending to higher-order differential equations of $x(t)$, the two equations are still valid, and they are

$$
\begin{aligned}
{\left[\begin{array}{cc}
x^{(2)} & x \\
x^{(3)} & x^{(1)}
\end{array}\right]\left[\begin{array}{l}
c_{1} \\
c_{2}
\end{array}\right] } & =-\left[\begin{array}{l}
x^{(4)} \\
x^{(5)}
\end{array}\right], \\
x^{(2)} c_{1}+x c_{2} & =-x^{(4)}, \\
x^{(3)} c_{1}+x^{(1)} c_{2} & =-x^{(5)} .
\end{aligned}
$$

Through (16), we obtain two expressions expressed by differential coefficient equations:

$$
\begin{aligned}
& c_{1}=-\frac{\dot{x} x^{(4)}-x x^{(5)}}{\dot{x} x^{(2)}-x x^{(3)}}=-\frac{\Upsilon_{5}(x)}{\Upsilon_{3}(x)}, \\
& c_{2}=\frac{x^{(2)} x^{(5)}-x^{(3)} x^{(4)}}{x x^{(3)}-\dot{x} x^{(2)}}=\frac{\Upsilon_{3}(\ddot{x})}{\Upsilon_{3}(x)} .
\end{aligned}
$$

The $k$-order continuous differential energy operator $\gamma_{k}[x]$ is defined as

$$
\Upsilon_{k}[x]=\dot{x} x^{(k-1)}-x x^{(k)} .
$$

So from the formula (18), we get the instantaneous frequency expression of the two signal components, expressed as

$$
w_{1,2}=\sqrt{\frac{c_{1} \pm \sqrt{c_{1}^{2}-4 c_{2}}}{2}} .
$$

Through the instantaneous frequencies and energy operators of the two signal components, instantaneous amplitudes of the signal components can be estimated:

$$
\begin{gathered}
\Psi_{c}\left[x^{(3)}\right]-w_{1}^{2} w_{2}^{2} \Psi_{c}[\dot{x}]=\left[a_{1}^{2} w_{1}^{6}-a_{2}^{2} w_{2}^{6}\right]\left[w_{1}^{2}-w_{2}^{2}\right] \\
\Psi_{c}[\ddot{x}]-w_{1}^{2} w_{2}^{2} \Psi_{c}[x]=\left[a_{1}^{2} w_{1}^{4}-a_{2}^{2} w_{2}^{4}\right]\left[w_{1}^{2}-w_{2}^{2}\right] .
\end{gathered}
$$

Expressions of the instantaneous amplitudes of the two signal components can be obtained from the above two equations, expressed as

$$
\begin{aligned}
a_{1,2}^{2}= & \left(w_{2,1}^{4}\left(\Psi_{c}\left[x^{(3)}\right]-w_{1}^{2} w_{2}^{2} \Psi_{c}[\dot{x}]\right)\right. \\
& \left.-w_{2,1}^{6}\left(\Psi_{c}[\ddot{x}]-w_{1}^{2} w_{2}^{2} \Psi_{c}[x]\right)\right) \\
& \times\left(w_{1}^{4} w_{2}^{4}\left(w_{1}^{2}-w_{2}^{2}\right)^{2}\right)^{-1}
\end{aligned}
$$

After obtaining instantaneous frequency and amplitude of each signal component for time, we make a reconstruction by using the instantaneous frequency and magnitude of each signal component. At last we get two original signal components of the mixed signal, realizing the separation of mixed signals which contains two signal components. Energy operator signal separation method is suitable for the detected signal whose amplitude and frequency change slowly, at each time or in a period of time. Its signal components can also be seen as a single-frequency signal.

2.2. The Simulation Results. To verify the energy demodulation algorithm can separate mixed signal which contains two signal components effectively, the simulation experiment selected a mixed signal containing two AM-FM signal components as a source signal, the signal model of the simulation expressed as

$$
\begin{aligned}
x(n)= & a_{1}(n) \cos \left(\int_{0}^{n} \Omega_{1}(m) d m\right) \\
& +a_{2}(n) \cos \left(\int_{0}^{n} \Omega_{2}(m) d m\right),
\end{aligned}
$$

in the expression

$$
\begin{array}{r}
\Omega_{i}(n)=\Omega_{c i}+\Omega_{m i} \cos \left[\Omega_{f i} n+\theta_{i}\right], \\
\Omega_{c i}=\frac{2 \pi f_{c i}}{f_{s}}, \quad \Omega_{f i}=\frac{2 \pi f_{f i}}{f_{s}}, \\
a_{i}(n)=1+k_{a i} \cos \left(\Omega_{a i} n+\beta_{i}\right), \\
\Omega_{a i}=\frac{2 \pi f_{a i}}{f_{s}}, \quad \Omega_{m i}=k_{f i} \cdot \Omega_{f i}, \\
i=1,2 .
\end{array}
$$

Before setting the parameters of the simulated signals, we defined several parameters which described the performance of the mixed signal containing two signal components. Parameter SEP measured the condition of spectrum separation between the signal components. A smaller SEP value indicated that the overlap degree of the spectrum between the components was greater, which was defined as

$$
\mathrm{SEP}=\frac{\left|\Omega_{c 1}-\Omega_{c 2}\right|}{\sum_{i}\left(\Omega_{f i}+\Omega_{a i}+\Omega_{m i}\right)}, \quad i=1,2 .
$$


TABLE 1: Parameters and performance parameters of simulated signal 1.

\begin{tabular}{lccc}
\hline $\begin{array}{l}\text { The parameter } \\
\text { name }\end{array}$ & $\begin{array}{c}\text { The parameter } \\
\text { values }\end{array}$ & $\begin{array}{c}\text { The parameter } \\
\text { name }\end{array}$ & $\begin{array}{c}\text { The parameter } \\
\text { values }\end{array}$ \\
\hline$f_{s}$ & $15 \mathrm{k}$ & SEP & 0.6250 \\
$f_{c 1}, f_{c 2}$ & 100,150 & CR/FD1 & 20.0 \\
$f_{a 1}, f_{a 2}$ & 30,20 & CR/FD2 & 15.0 \\
$f_{f 1}, f_{f 2}$ & 5,10 & CR/IB1 & 3.3 \\
$k_{f 1}, k_{f 2}$ & 1,1 & CR/IB2 & 5.0 \\
\hline
\end{tabular}

TABLE 2: Correlation coefficients of separated signal and the original signal.

\begin{tabular}{lcc}
\hline Correlation coefficient & $\begin{array}{c}\text { The obtained } \\
\text { signal } \times 1\end{array}$ & $\begin{array}{c}\text { The obtained } \\
\text { signal } \times 2\end{array}$ \\
\hline The original signal S1 & 0.9418 & 0.0843 \\
The original signal S2 & 0.2932 & 0.9484 \\
\hline
\end{tabular}

Parameter CR/IB measured the speed changes for the signal modulation relative to the carrier. The higher value of $\mathrm{CR} / \mathrm{IB}$ indicated the mixed signal was closer to a stable sinusoidal signal. It was defined as

$$
\frac{\mathrm{CR}}{\mathrm{IB}}=\frac{\Omega_{c i}}{\max \left(\Omega_{f i}, \Omega_{a i}\right)}, \quad i=1,2 .
$$

Parameter CR/FD measured the intensity of signal modulation. When CR/FD increased, the intensity of the modulation signal component reduced; on the contrary, a smaller $\mathrm{CR} / \mathrm{FD}$ indicates that the intensity of signal modulation was greater. It was defined as

$$
\frac{\mathrm{CR}}{\mathrm{FD}}=\frac{\Omega_{c i}}{\Omega_{m i}}, \quad i=1,2 .
$$

2.2.1. The Experimental Results of the Overlap Degree Less Than 1. The simulation used a mixed signal containing two signal components as the source signal to verify that the signal demodulation algorithm based on energy signal separation method could achieve single-channel blind signal separation, mixed signal model expressed as formula (22) [17]. Signal parameters and performance parameters defined by the values were shown in Table 1 . The sampling frequencies $f_{s}, f_{c 1}$, and $f_{c 2}$ were, respectively, the carrier frequencies of the two signal components, $f_{a 1}$ and $f_{a 2}$ were, respectively, the amplitude-modulated frequencies of signal 1 and signal $2, f_{f 1}$ and $f_{f 2}$ were, respectively, the frequencies of signal 1 and signal 2, and $k_{f 1}$ and $k_{f 2}$ were, respectively, the frequency modulation factors of the two signals.

Figure 1 showed the mixed signal waveform and frequency spectrum of two signal components. Seen from Table 1, the normalized mixed signal carrier frequency ratio SEP was 0.6250. By using the multicomponent signal separation method based on energy demodulation algorithm, we separated signals and reconstructed the two signals and then calculated the correlation coefficient of the original signal, as shown in Table 2.
TABLE 3: Parameters and performance parameters of simulated signal 2 .

\begin{tabular}{lccc}
\hline $\begin{array}{l}\text { The parameter } \\
\text { name }\end{array}$ & $\begin{array}{c}\text { The parameter } \\
\text { values }\end{array}$ & $\begin{array}{c}\text { The parameter } \\
\text { name }\end{array}$ & $\begin{array}{c}\text { The parameter } \\
\text { values }\end{array}$ \\
\hline$f_{s}$ & $15 \mathrm{k}$ & SEP & 1.0204 \\
$f_{c 1}, f_{c 2}$ & 130,80 & $\mathrm{CR} / \mathrm{FD} 1$ & 32.5 \\
$f_{a 1}, f_{a 2}$ & 10,12 & $\mathrm{CR} / \mathrm{FD} 2$ & 16.0 \\
$f_{f 1}, f_{f 2}$ & 5,10 & $\mathrm{CR} / \mathrm{IB} 1$ & 13.0 \\
$k_{f 1}, k_{f 2}$ & $0.5,0.5$ & $\mathrm{CR} / \mathrm{IB} 2$ & 8.0 \\
\hline
\end{tabular}

TABLE 4: Correlation coefficients of acquired signal and the original signal.

\begin{tabular}{lcc}
\hline Correlation coefficient & $\begin{array}{c}\text { The obtained } \\
\text { signal } \times 1\end{array}$ & $\begin{array}{c}\text { The obtained } \\
\text { signal } \times 2\end{array}$ \\
\hline The original signal S1 & 0.9873 & 0.0996 \\
The original signal S2 & 0.0692 & 0.9794 \\
\hline
\end{tabular}

A comparison waveform diagram of acquired signal and the original signal was shown in Figure 2. As can be seen from the Figure 2, the acquired signal components were very close to the original signals, and the correlation coefficient of the original signal component 1 and the acquired signal 1 was 0.9418 , and the correlation coefficient of the original signal component 2 and the acquired signal 2 was 0.9484 . Errors only appear in some peaks and troughs, which might be due to that signals changed fast at peaks and troughs.

2.2.2. The Experimental Results of the Overlap Degree More Than 1. The simulation used a mixed signal containing two signal components as source signal, mixed signal model shown as the formula (22). Signal parameters and performance parameters defined by the values were shown in Table 3. The sampling frequencies $f_{s}, f_{c 1}$, and $f_{c 2}$ were, respectively, the carrier frequencies of the two signal components, $f_{a 1}$ and $f_{a 2}$ were, respectively, the amplitudemodulated frequencies of signal 1 and signal $2, f_{f 1}$ and $f_{f 2}$ were, respectively, the frequencies of signal 1 and signal 2 , and $k_{f 1}$ and $k_{f 2}$ were, respectively, the frequency modulation factors of the two signals.

Figure 3 showed the signal waveform and frequency spectrum of a mixed signal containing two components. Table 3 showed that the mixed signal normalized carrier frequency ratio parameter SEP was 1.0204, and signal aliasing extent was less than the above simulation A shown. Similarly, by using the separation method based on energy demodulation algorithm, we separated and reconstruct the two signal components and then calculated the correlation coefficient with the original signal, as shown in Table 4.

A comparison waveform diagram of acquired signal and the original signal was shown in Figure 4. As can be seen from Figure 4, the acquired signal components were very close to the original signals, and the correlation coefficient [18-20] of the original signal component 1 and the acquired signal 1 was 0.9873 , and the correlation coefficient of the original signal component 2 and the acquired signal 2 was 0.9794 . Compared 


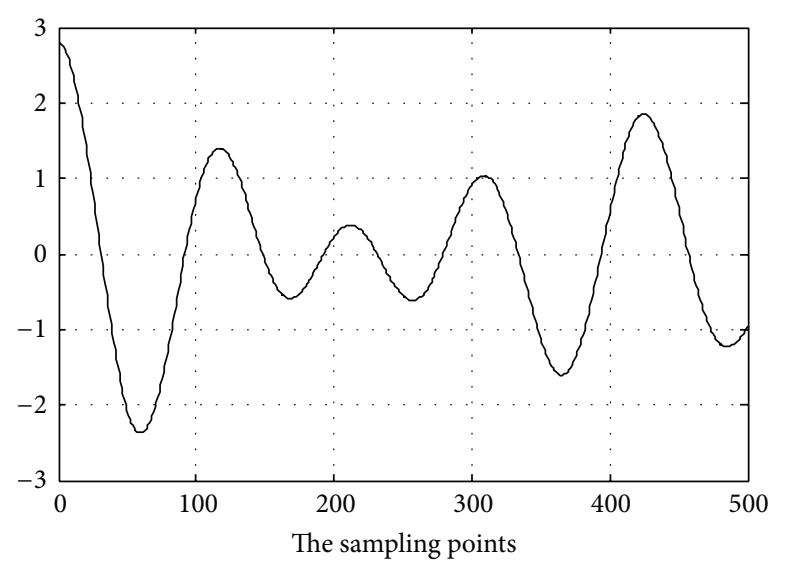

(a) Mixed signal waveform

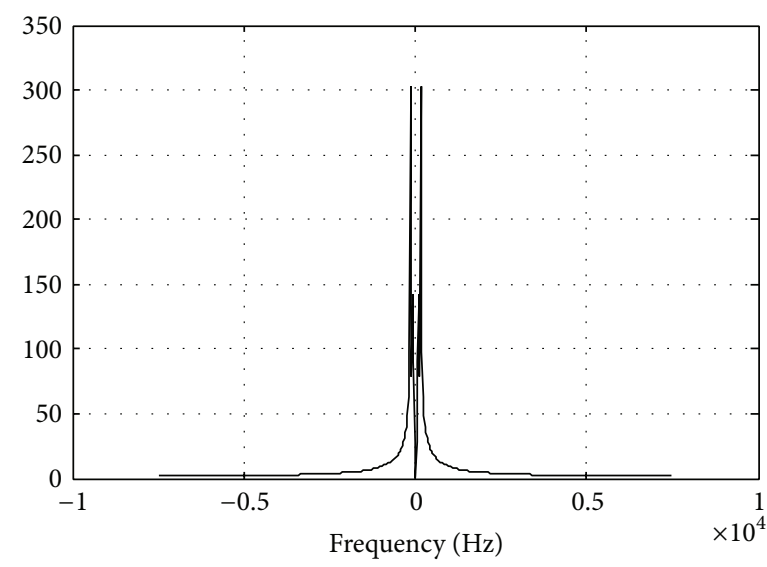

(b) Mixed signal spectrum

FIGURE 1: Mixed signal waveform and its spectrum.

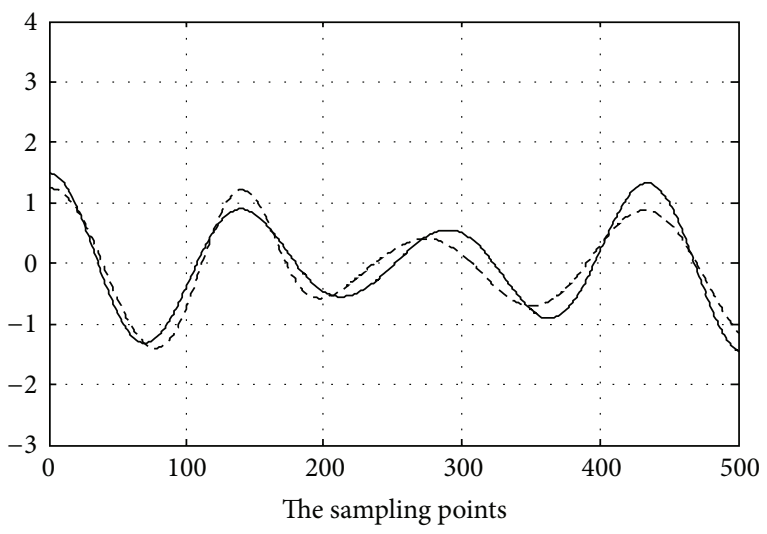

- Original signal 1

- - Reconstructed signal 1

(a) A comparison chart of signal 1

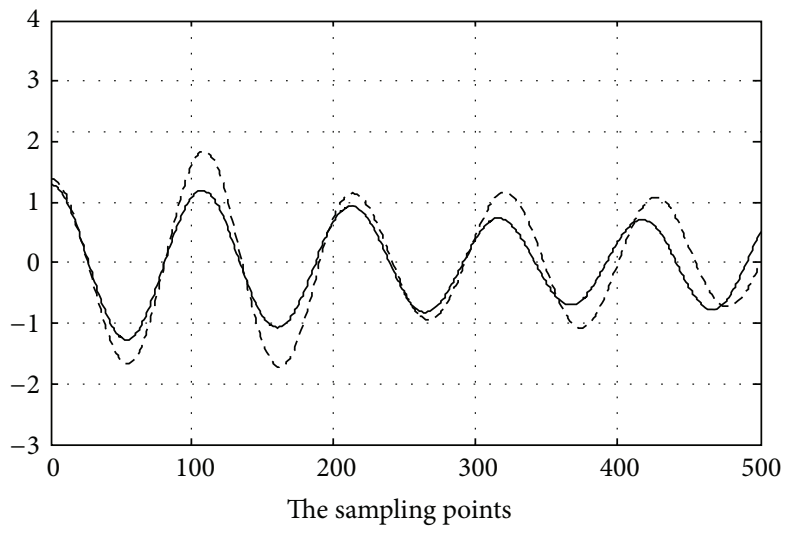

- Original signal 2

- - - Reconstructed signal 2

(b) A comparison chart of signal 2

FIGURE 2: The comparison chart for the reconstructed signal and original signal.

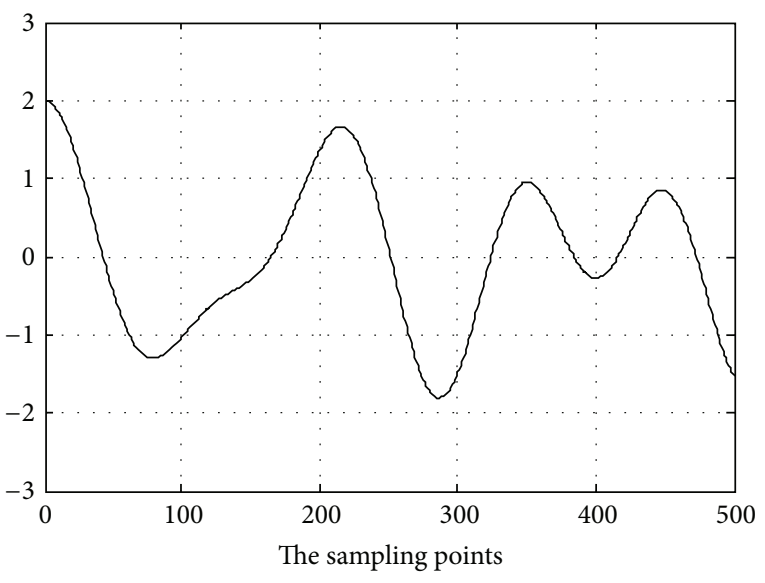

(a) Mixed signal waveform

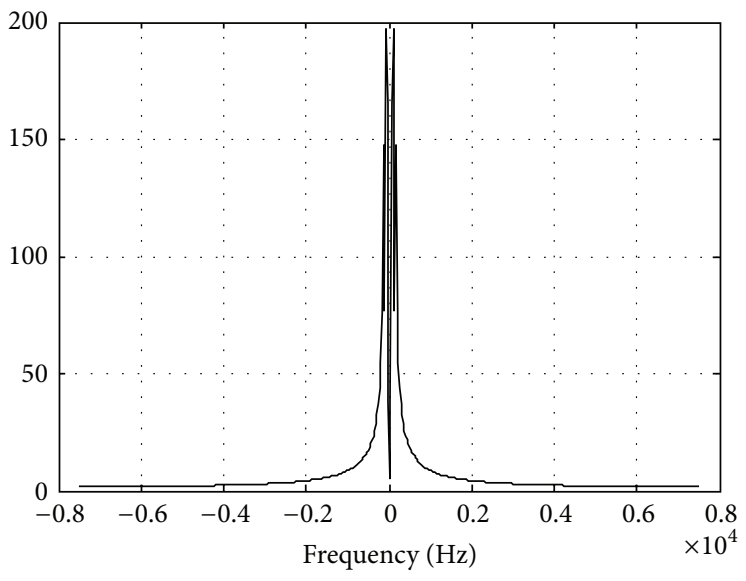

(b) Mixed signal spectrum

FIGURE 3: Mixed signal waveform and its spectrum. 


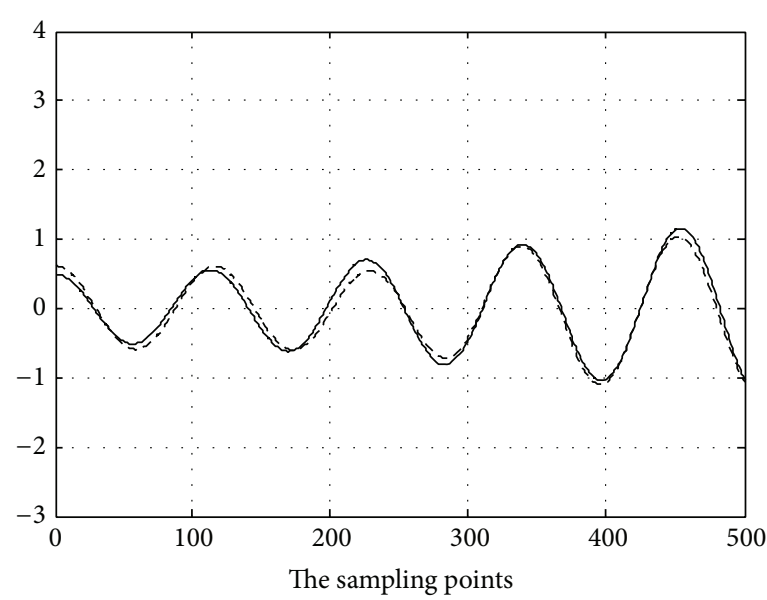

- Original signal 1

- - - Reconstructed signal 1

(a) A comparison chart of signal 1

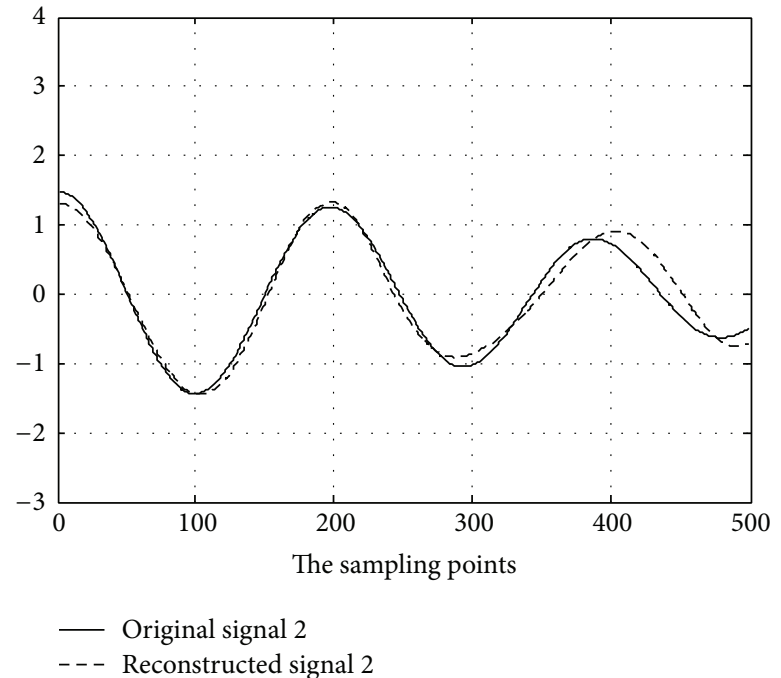

(b) A comparison chart of signal 2

FIGURE 4: The comparison chart for the reconstructed signal with the original signal.

to the results of simulation $\mathrm{A}$, the larger the normalized carrier frequency parameter, the better the separation. The greater the normalized carrier frequency parameter was, the smaller the degree of aliasing was, and the better the result of the separation was, by using the separation method based on energy demodulation algorithm.

Through the above two simulations, by using the separation method based on energy demodulation algorithm, good estimated results could be got. The separation of multicomponent signal provided a theoretical basis for the oil-gas-water flow velocity measurement.

\section{Gas-Liquid Velocity Measurement of the Oil-Gas-Water Three-Phase Flow}

3.1. The Superposition Characteristic for Single-Channel OilGas-Water Signal. When the oil-gas-water mixture flows through conductance sensor, due to the size distribution and the spatial distribution of oil bubble particles, the fluid conductivity changes randomly, and oil-gas-water mixed fluid flowing noise forms [21-23]. Under the effect of the excitation current, the measuring electrode of conductance sensor will detect water flow noise, so that we get the conductance sensor output signals which characterize the fluid flowing information. When the oil-gas-water threephase mixed liquid flows through the conductance sensors, it collects the flow noise signal with a frequency of $16 \mathrm{Khz}$, the sampling time is $62.5 \mu \mathrm{s}$, the length of the experimental data is 512 sampling points, and the total length of time for every frame of experimental data is $32 \mathrm{~ms}$. Figure 5 shows a six electrode conductance sensor, which consists of a pair of outer excitation electrodes and two rings of the measurement electrodes.

In the condition of water as continuous phase, when the mixture flows through the conductance sensor, the insulating oil bubbles cause the changes of electric field inside the electric conductance sensor [24]. As the oil bubble can be in any position inside the sensor, through modeling three-dimensional conductance sensor, we get electric-field distribution inside the conductance sensor which contains inhomogeneous media. We acquire the condition in which oil bubble is in different positions in the electric-field distribution inside the conductance sensor and the conductance sensor response characteristic when the oil bubbles move along the central axis of the sensor. Through the analysis of different number of oil bubbles which move different distances from the central axis of the sensor, we get the conductance sensor response characteristics which provide theoretical basis for the following oil-gas-water three-phase flow gas-liquid phase separation method. When the fluid flows through the conductance sensor, the measured conductance sensor rings have no hindrance effect on the fluid flow. The conductance sensor collects the flow noise signal with a frequency of $16 \mathrm{KHz}$, and the fluid flow pattern does not change.

Li et al. [25] proved the signal characteristics of one bubble in the conductance sensor in detail. Li et al. [26] explained why the mixed signal contained the oil bubble and gas bubble flow noise signals together. In this paper, our study is based on the mixed signal which is composed of two wave noise signals. When the bubbles move along the axis direction, the superposition of two oil bubble signals is equal to the conductance sensor signal which contains two bubbles in conductance sensor at the same time along the axis direction, which proves that the conductance sensor satisfies the superposition theorem. Figure 6 proves the conductance sensor satisfies the superposition theorem. Figure 6(a) represents the superposition of two independent bubble signals. Figure 6(b) shows the comparison of experimental and superposition values. Experimental value represents a conductance sensor collection value which contains two bubbles moving through it. Superposition value is the superposition of two collection values which contain only one bubble. 


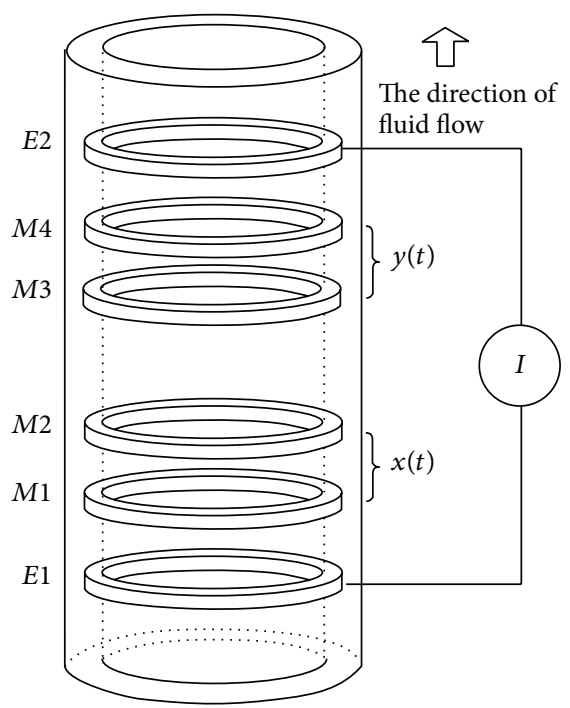

(a) Longitudinal multielectrode conductance sensor

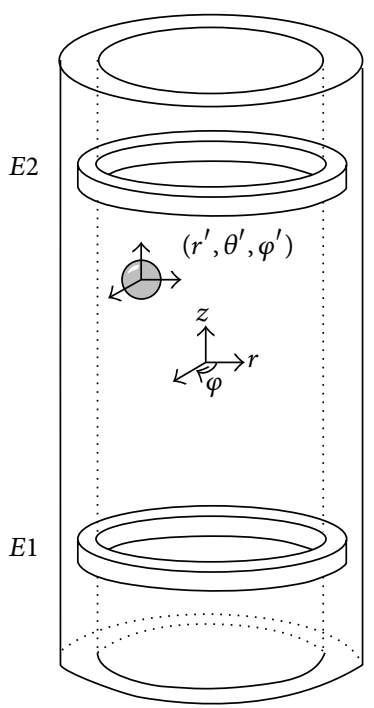

(b) 3D model of conductance sensor

FIGURE 5: Longitudinal multipole conductance sensor model.

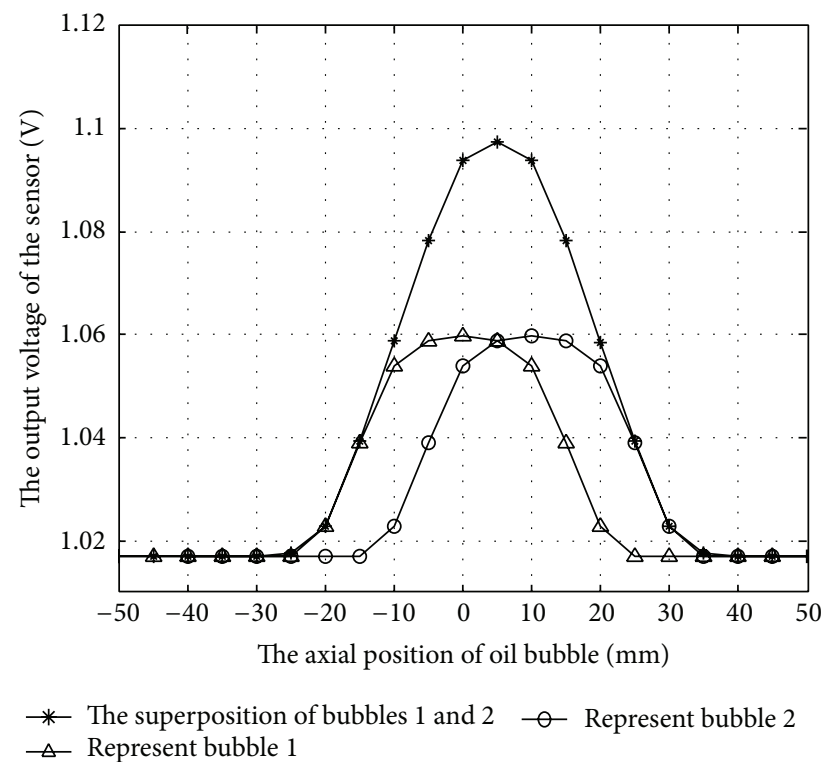

(a) Superposition of two independent bubble signals

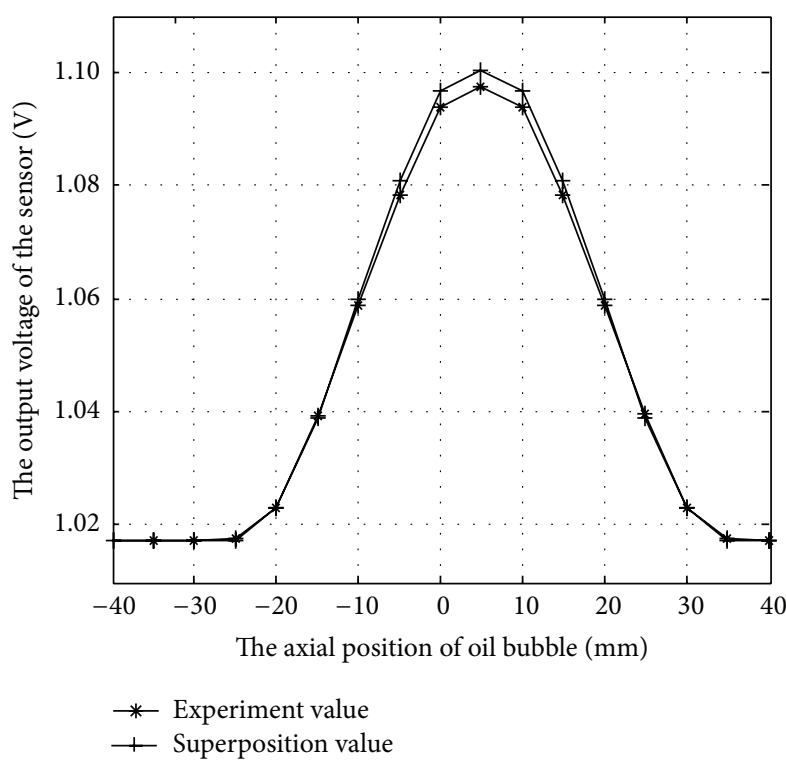

(b) Comparison of experimental and superposition values

FIGURE 6: The superposition theorem of conductance sensor.

By means of analyzing the conductance sensor response superposition characteristics, the signal is actually a mixed signal which contains two flowing noise signal components, of which one is generated by the gas-phase flow and the other is generated by the oil-phase flow. By using correlation measurement technique, we can conduct the measurements of velocity $[27,28]$.

\subsection{Single-Channel Oil-Gas-Water Gas-Liquid Velocity Mea-} surement Process. The vertical multielectrode conductance sensor measuring electrode collects oil-gas-water threephase flow noise signal. The signal is a mixed signal which combines the gas-phase and the oil-phase flow noise signal components together. Signal separation method based on energy demodulation algorithm can be implemented to demodulate the signal containing two components. We can get a gas-water fluctuation signal and an oil-water fluctuation signal. Then to get the gas-phase velocity and liquid-phase velocity, we calculate the delay time of the gas phase and liquid phase by using the correlation method. Flow velocity measurement principle of the oil-gas-water three-phase flow is shown in Figure 7.

Firstly, vertical multielectrode conductance sensor collected oil-gas-water three-phase flow noise signal. After 


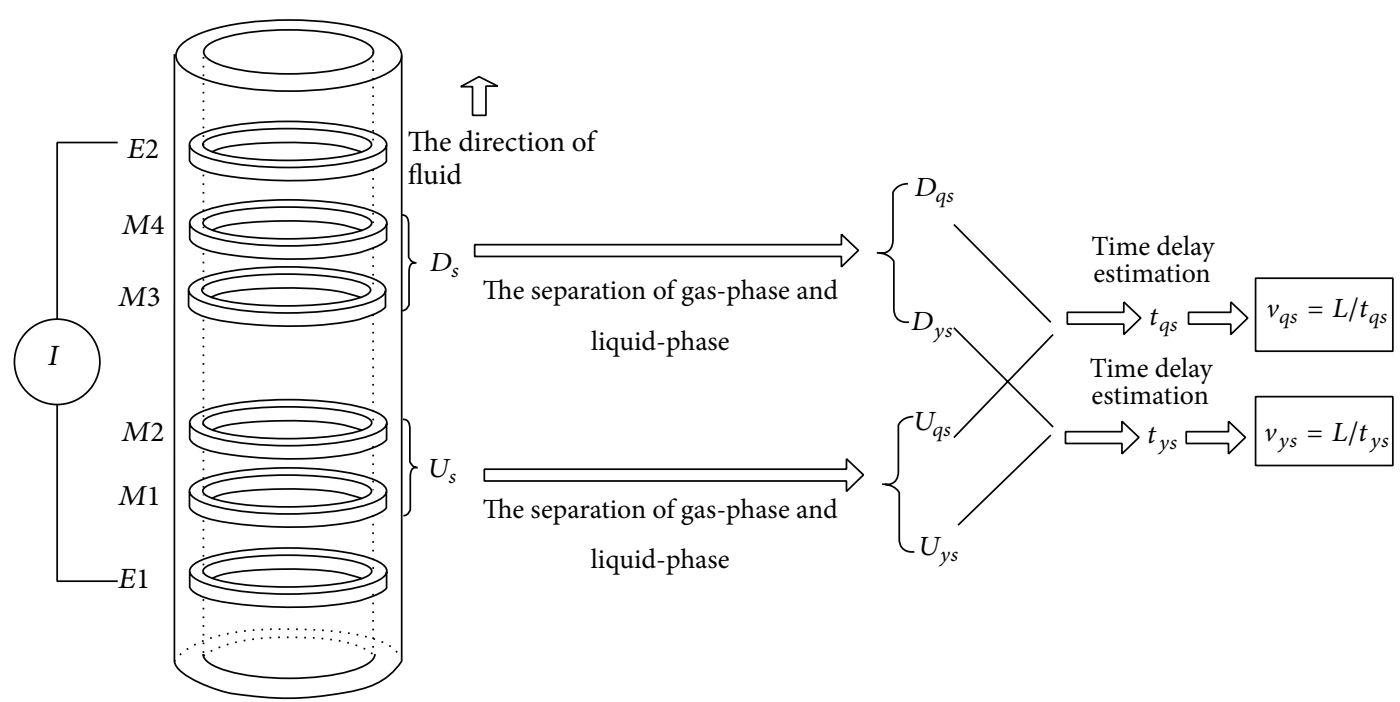

FIGURE 7: Principle for gas-liquid phase velocity measurement of the oil-gas-water three-phase flow.

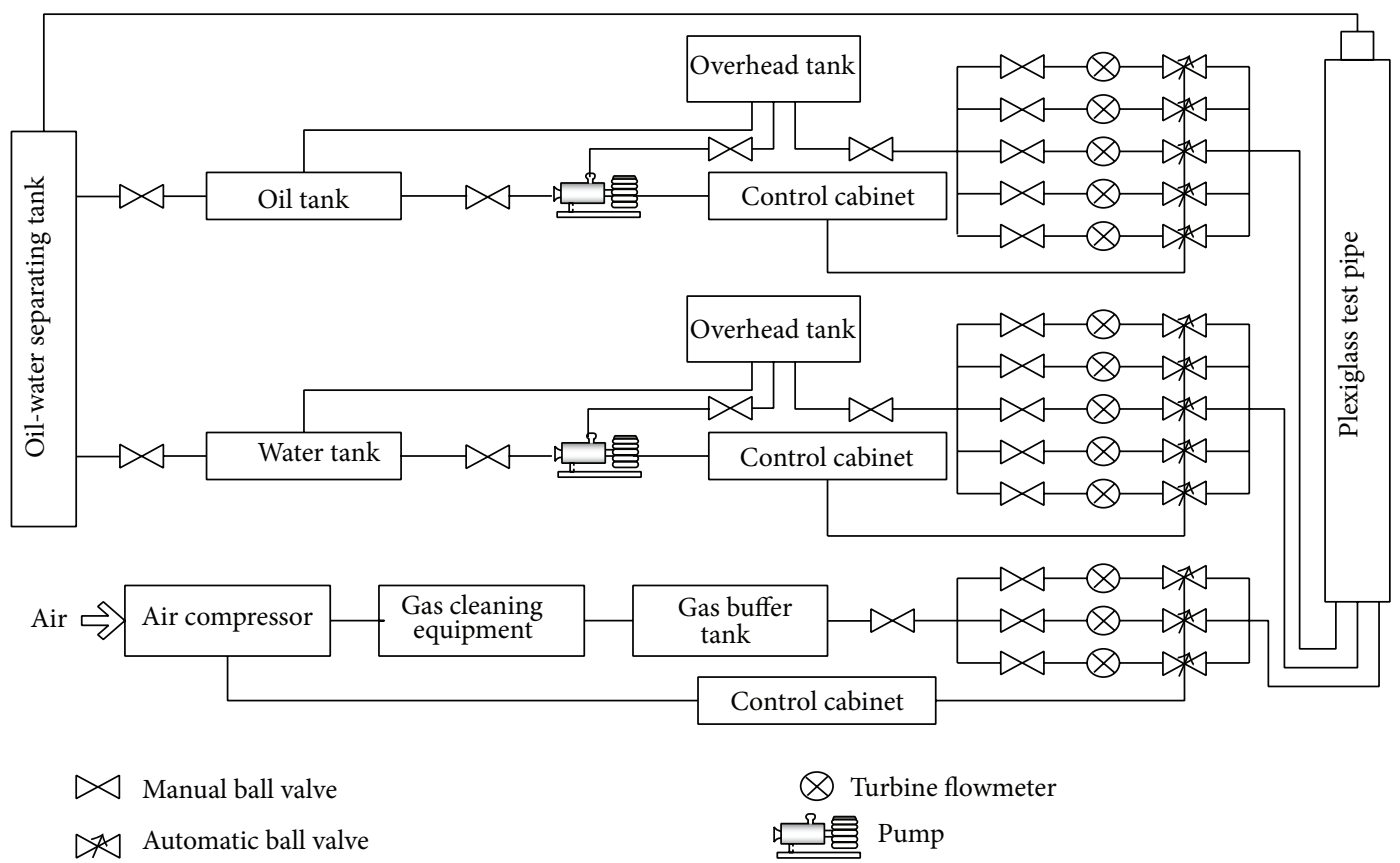

FiguRE 8: The circulation schematic diagram of oil-gas-water three-phase flow loop.

sampling, the oil-gas-water three-phase flow signal could be expressed as $x(n)$. According to the single-frequencysignal hypothesis, oil-gas-water three-phase flow signal $x(n)$ satisfied the differential equation

$$
\begin{gathered}
c_{1}[x(n-1)+x(n-3)]+c_{2} x(n-2) \\
+[x(n)+x(n-4)]=0 .
\end{gathered}
$$

The differential equation coefficients $c_{1}, c_{2}$, respectively, were

$$
\begin{gathered}
c_{1}=-2\left(\cos \Omega_{1}+\cos \Omega_{2}\right), \\
c_{2}=4 \cos \Omega_{1} \cos \Omega_{2}+2 .
\end{gathered}
$$

$\Omega_{1}, \Omega_{2}$ were instantaneous frequencies of the two components of the oil-gas-water three-phase flow noise signal.

By using the energy operator and differential energy operator, differential equation coefficients could be estimated:

$$
\begin{aligned}
c_{1}= & \frac{\Upsilon_{3}[x(n-3)]-\Upsilon_{3}[x(n-1)]}{\Psi[x(n-1)]-\Psi[x(n-2)]}, \\
c_{2}= & (\Psi[x(n)]-\Psi[x(n-3)] \\
& \left.+\Upsilon_{4}[x(n-2)]-\Upsilon_{4}[x(n-3)]\right) \\
& \times(\Psi[x(n-1)]-\Psi[x(n-2)])^{-1} .
\end{aligned}
$$




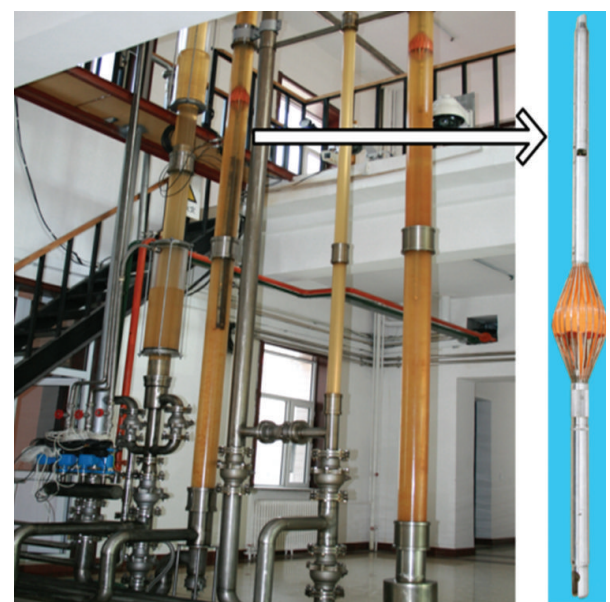

Figure 9: The equipment of multiphase-flow experimental equipment and conductance correlation flowmeter.

In the equations, $\Psi[x(n)]$ was the energy operator of oil-gas-water three-phase flow noise signal $x(n)$, and its expression was $\Psi[x(n)]=x^{2}(n)-x(n-1) x(n+1), \gamma_{k}[x(n)]$ was the $k$-order discrete difference energy operator of the oil-gas-water three-phase flow noise signal $x(n): \gamma_{k}[x(n)]=$ $x(n) x(n+k-2)-x(n-1) x(n+k-1)$. By using (24) and (25), we could get the oil-gas-water three-phase flow noise signals instantaneous frequencies of the two signal components:

$$
\Omega_{1,2}=\arccos \left(-\frac{c_{1}}{4} \pm \frac{\sqrt{c_{1}^{2}-4 c_{2}+8}}{4}\right) .
$$

Then we used the obtained instantaneous frequencies, symmetric differential and energy operator functions of the two signal components of oil-gas-water three-phase flow noise signals to get the instantaneous amplitudes of the two signal components and the estimation formula:

$$
\begin{aligned}
a_{1,2}^{2}= & \left(S_{2,1}^{4}\left[\Psi\left[\Delta_{s}^{3} x\right]-S_{1}^{2} S_{2}^{2} \Psi\left[\Delta_{s} x\right]\right]\right. \\
& \left.-S_{2,1}^{6}\left[\Psi\left[\Delta_{s}^{2} x\right]-S_{1}^{2} S_{2}^{2} \Psi[x]\right]\right) \\
& \times\left(S_{1}^{4} S_{2}^{4}\left[S_{1}^{2}-S_{2}^{2}\right]^{2}\right)^{-1} .
\end{aligned}
$$

And $S_{1,2}=\sin \left(\Omega_{1,2}\right), \Delta_{s} x=[x(n+1)-x(n-1)] / 2$, besides $\Delta_{s}^{m} x=\Delta_{s}\left(\Delta_{s}^{m-1} x\right), \Psi\left[\Delta_{s}^{n} x\right]=\Psi\left[\Delta_{s}^{m} x, \Delta_{s}^{n} x\right]$. The definition for $\Psi\left[\Delta_{s}^{m} x, \Delta_{s}^{n} x\right]$ is $\Psi\left[\Delta_{s}^{m} x, \Delta_{s}^{n} x\right]=\Delta_{s}^{m} x(n) \cdot \Delta_{s}^{n} x(n)-\Delta_{s}^{m} x(n-$ 1) $\cdot \Delta_{s}^{n} x(n+1)$.

According to the instantaneous frequencies and the instantaneous amplitudes of the two signal components, we could reconstruct the two signal components, so that by using signal separation method based on energy demodulation algorithm we could get the two signal components of oilgas-water three-phase flow noise signals. By using the above method, we separated upstream oil-gas-water three-phase flow noise signal $U_{s}$ and downstream oil-gas-water threephase flow noise signal $D_{s}$ which were all collected from the vertical multielectrode conductance sensor. And after the gas-liquid phase identification, we could get the upstream gas-water fluctuation signal $U_{q s}$ and oil-water fluctuation signal $U_{y s}$, the downstream gas-water fluctuation signal $D_{q s}$ and oil-water fluctuation signal $D_{y s}$.

Then by using the correlation method, we obtained the time delay $t_{q s}$ of the upstream gas-water fluctuation signals and downstream gas-water fluctuation signal, as well as $t_{y s}$ of the upstream oil-water fluctuation signals and downstream oil-water fluctuation signal:

$$
\begin{aligned}
& v_{q s}=\frac{L}{t_{q s}}, \\
& v_{y s}=\frac{L}{t_{y s}} .
\end{aligned}
$$

Through (32), we calculated the gas-water two-phase flow velocity $V_{q s}$ and oil-water two-phase flow velocity $V_{y s}$. $L$ was the distance between the upstream and downstream signals, so that we achieved the measurements of oil-gas-water threephase flow gas-phase velocity and liquid-phase velocity.

The algorithm is a combination of energy operator and correlation measurement technique. This algorithm is a method researched in the bubbly flow which has low flow and less bubbles. Its main advantage is that in a short period of time, by using less data, the instantaneous flow velocity can be figured out, and the measured results have good real-time characteristics in the range of allowable error.

3.3. Gas-Liquid Velocity Measurement Results of the Oil-GasWater Three-Phase Flow. The experiments were carried out on a three-phase flow simulation test device. The device consists of a transparent plexiglass test pipe with 8 meters in length and 125 millimeters' inner diameter, an oil-water separating tank, a buffer tank, two overhead tanks, several control systems, and other components [29]. The schematic diagram of oil-gas-water flow loop is shown in Figure 8. In the experiments, the experimental fluids were made of diesel oil, air, and water, the liquid pattern was bubbly flow, and the velocity was less than $30 \mathrm{~m}^{3} / \mathrm{d}$. The whole experiment was established based on the bubbly flow. Air was supplied from an air compressor and flowed through a gas cleaning equipment into a gas buffer tank. Oil and water were supplied from an oil tank and a water tank and flowed into an oil overhead tank and a water overhead tank through oil pump and water pump, respectively. Then air, oil, and water from the buffer tank or the overhead tanks flowed into the plexiglass test pipe after being measured by the turbine flowmeter, and they streamed from bottom to top. Air from the test pipe was discharged directly. The oil-water mixture was put into the oil-water separating tank. After gravity separation, oil and water flowed into the oil tank and the water tank, respectively, for cycling utilization.

The experiments used a conductance correlation flowmeter with 20 millimeters' inner diameter. It is made up of a fan current collector, a six electrode conductivity sensor, a drive circuit system, and so forth. In the experiments, the measuring instrument was put into the transparent plexiglass test pipe, and the fan current collector was opened. When the 


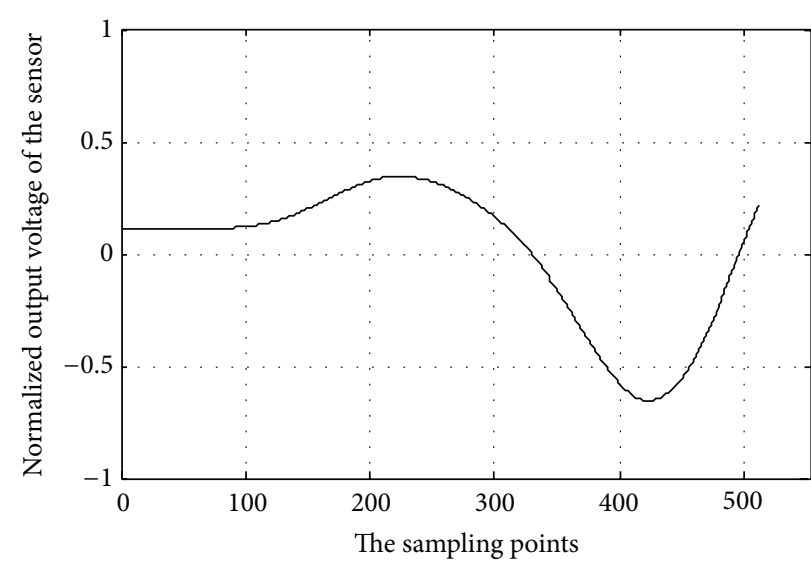

(a) The upstream oil-gas-water flow noise signal $U_{s}$

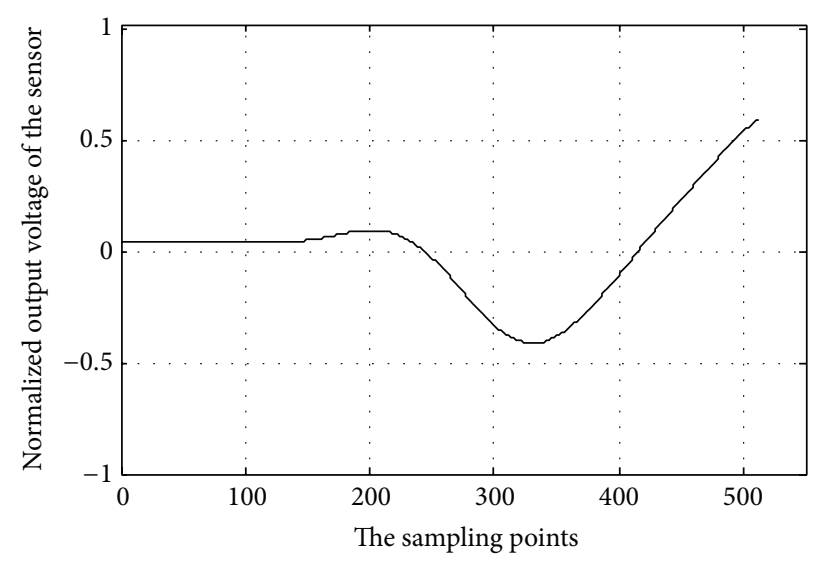

(b) The downstream oil-gas-water flow noise signal $D_{s}$

FIGURE 10: Upstream and downstream oil-gas-water three-phase flow noise signal.

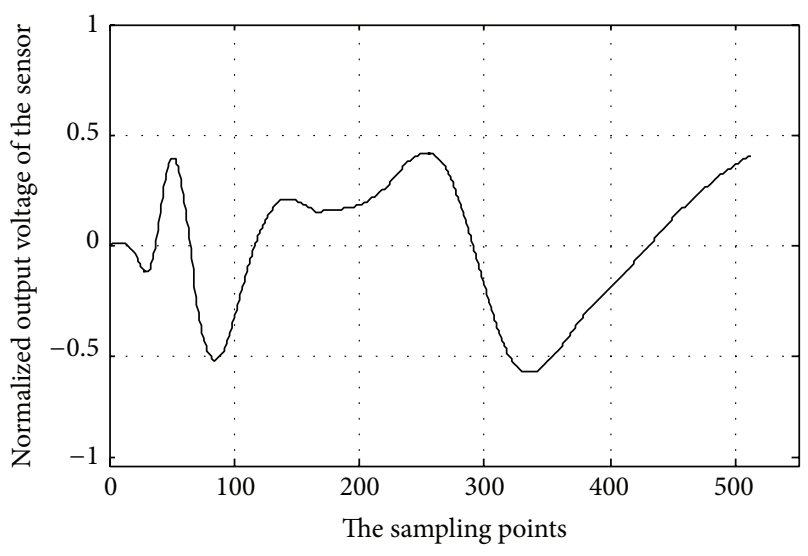

(a) The upstream gas-water fluctuation signal $U_{q s}$

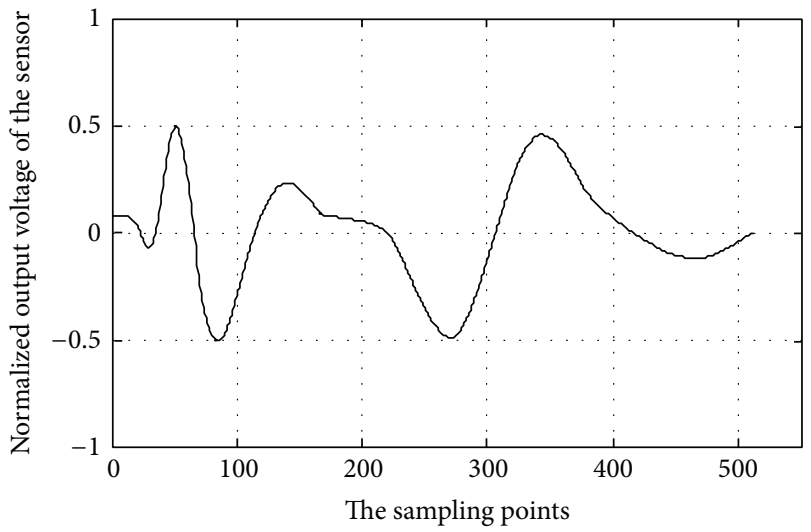

(c) The downstream gas-water fluctuation signal $D_{q s}$

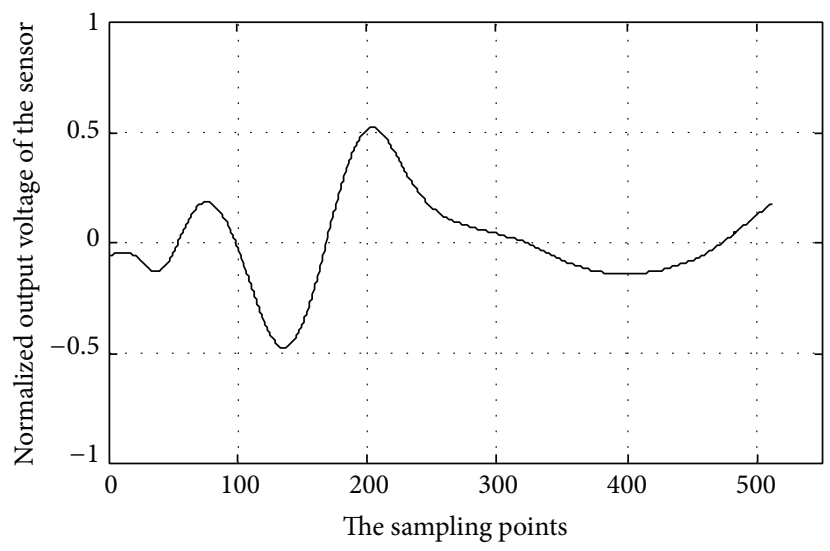

(b) The upstream oil-Water fluctuation signal $U_{y s}$

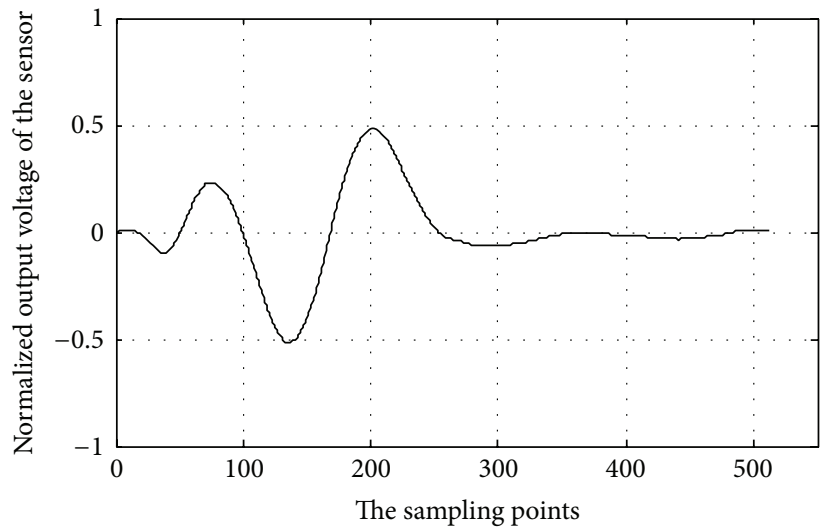

(d) The downstream oil-water fluctuation signal $D_{y s}$

FIGURE 11: The acquired upstream and downstream gas-water and oil-water fluctuation signals.

conductive oil-gas-water three-phase fluid was flowing from the sensor, upstream and downstream sections of the conductivity sensor were sensitive to the electrical conductivity of the mixed fluid. And the fluctuation signal changing with the electrical conductivity of the mixed fluid was the output. The physical maps of the multiphase-flow experimental equipment and conductance correlation flowmeter are shown in Figure 9.

Discrete phase oil bubbles and water bubbles distribute in the water. The oil-gas-water three-phase flow mixed 


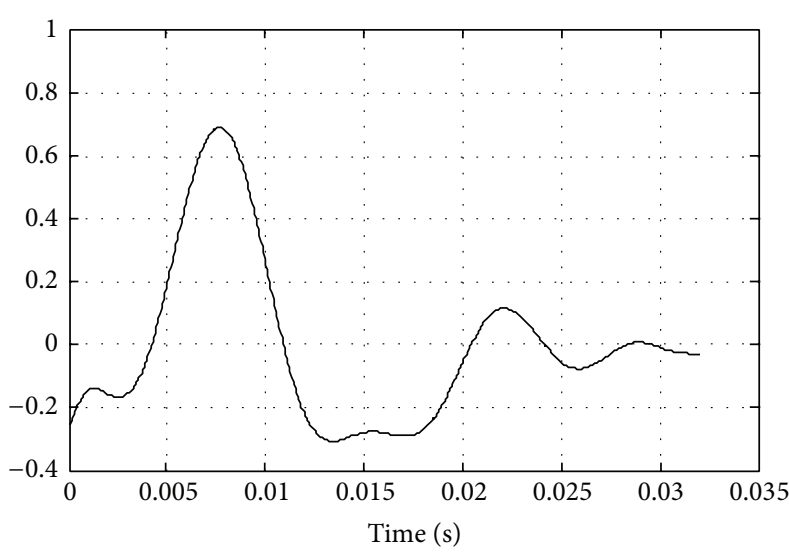

(a) Correlation function curve of gas-water fluctuation signal

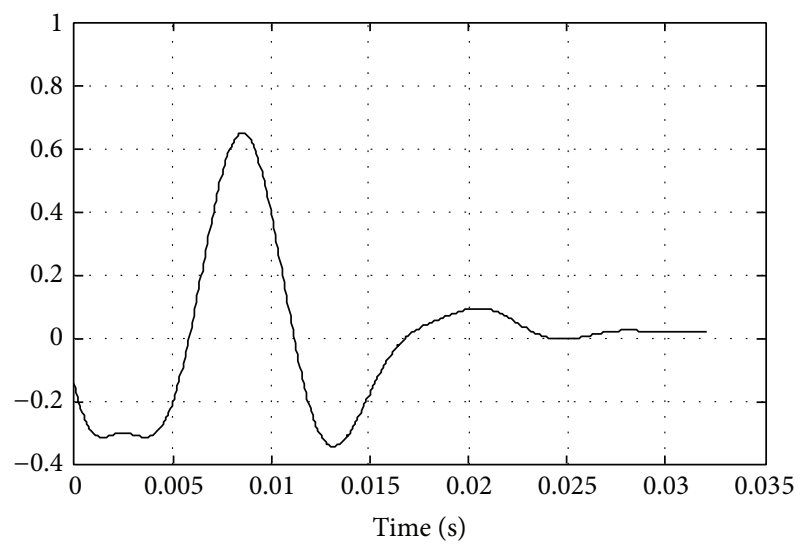

(b) Correlation function curve of oil-water fluctuation signal

FIGURE 12: Gas-water and oil-water fluctuation signal correlation function curves.

fluid has become uniform fluid before flowing into conductance sensor. Figure 10(a) showed the upstream oilgas-water three-phase flow noise signal, and Figure 10(b) showed the downstream oil-gas-water three-phase flow noise signal.

The upstream flow noise signal $U_{s}$ and the downstream flow noise signal $D_{s}$ were separately demodulated by oil-gaswater three-phase flow gas-liquid phase separation method which was based on energy demodulation algorithm. After that we got the upstream gas-water fluctuation signal $U_{q s}$ and oil-water fluctuation signal $U_{y s}$, the downstream gas-water fluctuation signal $D_{q s}$ and oil-water fluctuation signal $D_{y s}$. The results were shown in Figures 11(a)-11(d).

After the correlation method, we figured out the estimation time delay of upstream air-water fluctuation signals $U_{q s}$ and downstream gas-water fluctuation signal $D_{q s}$, as well as the estimation time delay of the upstream and downstream oil-water fluctuation signals. From Figure 12 we could get the time delay of the gas-water fluctuation signal and oil-water fluctuation signal. Figure 12(a) showed the relevant function curve of upstream gas-water fluctuation signal and downstream gas-water fluctuation signal; Figure 12(b) showed the relevant function curve of upstream oil-water fluctuation signal and downstream oil-water fluctuation signal.

To further verify that this method could achieve oilgas-water three-phase flow gas-liquid phase velocity measurement, we used the total flow rate $10 \mathrm{~m}^{3} / \mathrm{d}, 15 \mathrm{~m}^{3} / \mathrm{d}$, $20 \mathrm{~m}^{3} / \mathrm{d}, 30 \mathrm{~m}^{3} / \mathrm{d}$ of oil-gas-water three-phase flow liquid to measure gas-phase velocity and liquid-phase velocity. "10olg1w8" represented that the flow rate of the total oilgas-water three-phase flow was $10 \mathrm{~m}^{3} / \mathrm{d}$, gas flow rate was $1 \mathrm{~m}^{3} / \mathrm{d}$, oil flow rate was $1 \mathrm{~m}^{3} / \mathrm{d}$, and water flow rate was $8 \mathrm{~m}^{3} / \mathrm{d}$. The unit of gas-liquid phase velocities was $10^{-1} \mathrm{~m} / \mathrm{s}$. The measurement results were shown in Table 5.

As can be seen from Table 5, we used the oil-gas-water three-phase flow gas-liquid separation method which was based on energy demodulation algorithm to demodulate the upstream and downstream oil-gas-water three-phase flow fluctuation noise signals and then used the correlation method to get the gas velocity and liquid velocity. By this method, the obtained gas velocity and liquid velocity were very close to the actual values, and the measurement error was less than $10 \%$, which indicated that oil-gas-water threephase flow gas-liquid flow velocity measurement method based on the energy demodulation algorithm could effectively achieve the oil-gas-water three-phase flow gas-phase velocity and liquid-phase velocity.

The method is a combination of the energy demodulation algorithm and related measurement method for the instantaneous velocity experiment of oil-gas-water threephase flow. The conductance sensor sampling frequency is $16 \mathrm{Khz}$. According to the demand of instantaneous velocity, this experiment chooses a frame of 512 points as experimental data; the total time of experimental data is $32 \mathrm{~ms}$. If choosing a big experimental data in the algorithm, the signal would contain multiple oil bubble energy features, and the signal energy structure is complex, which will bring algorithm error, and the accuracy of velocity measurement results will become worse.

\section{Conclusion}

This paper used the oil-gas-water three-phase flow gasliquid phase separation method which was based on energy demodulation algorithm to demodulate the oil-gas-water three-phase flow signal which was collected by conductance sensor. After obtaining upstream gas-water fluctuation signal and oil-water fluctuation signal, downstream gas-water fluctuation signal and oil-water fluctuation signal, we figured out the gas velocity and liquid velocity by using the correlation method. The experimental results showed that this method could not only realize the oil-gas-water three-phase flow gasliquid phase velocity measurement, and the measurement error met the needs, but also proved that when the oil-gaswater three-phase flow liquid flows through the conductance sensor, the response characteristics met the superposition theorem. 
TABle 5: The comparison of experimental values and the actual values for oil-gas-water three-phase flow gas velocity and liquid velocity.

\begin{tabular}{|c|c|c|c|c|c|c|}
\hline Condition & Experiment value & $\begin{array}{l}\text { The gas velocity } \\
\text { actual value }\end{array}$ & Measurement error & Experiment value & $\begin{array}{l}\text { The oil velocity } \\
\text { actual value }\end{array}$ & Measurement error \\
\hline 10olg1w8 & 0.378 & 0.368 & $2.72 \%$ & 0.336 & 0.368 & $8.69 \%$ \\
\hline 10olg2w7 & 0.743 & 0.737 & $0.81 \%$ & 0.375 & 0.368 & $1.90 \%$ \\
\hline $10 \mathrm{o} 2 \mathrm{~g} 2 \mathrm{w} 6$ & 0.698 & 0.737 & $5.29 \%$ & 0.755 & 0.737 & $2.44 \%$ \\
\hline $1503 g 3 w 9$ & 1.124 & 1.105 & $1.72 \%$ & 1.072 & 1.105 & $2.98 \%$ \\
\hline $1503 g 6 w 6$ & 2.194 & 2.211 & $0.77 \%$ & 1.181 & 1.105 & $6.88 \%$ \\
\hline 15o4glw10 & 0.387 & 0.368 & $5.16 \%$ & 1.355 & 1.474 & $8.07 \%$ \\
\hline $1504 g 3 w 8$ & 1.152 & 1.105 & $4.25 \%$ & 1.589 & 1.474 & $7.80 \%$ \\
\hline $20 \mathrm{o} 2 \mathrm{~g} 2 \mathrm{w} 16$ & 0.768 & 0.737 & $4.20 \%$ & 0.755 & 0.737 & $2.44 \%$ \\
\hline $20 \mathrm{o} 4 \mathrm{~g} 6 \mathrm{w} 10$ & 2.304 & 2.211 & $4.21 \%$ & 1.589 & 1.474 & $7.80 \%$ \\
\hline $20 \mathrm{o} 6 \mathrm{~g} 2 \mathrm{w} 12$ & 0.668 & 0.737 & $9.36 \%$ & 2.304 & 2.210 & $4.25 \%$ \\
\hline $20 \mathrm{o} 6 \mathrm{~g} 6 \mathrm{w} 8$ & 2.304 & 2.210 & $4.25 \%$ & 2.194 & 2.211 & $0.77 \%$ \\
\hline $20 \mathrm{o} 8 \mathrm{~g} 2 \mathrm{w} 10$ & 0.688 & 0.737 & $6.65 \%$ & 2.880 & 2.948 & $2.31 \%$ \\
\hline $30 \mathrm{o} 2 \mathrm{~g} 4 \mathrm{w} 24$ & 1.440 & 1.474 & $2.31 \%$ & 0.781 & 0.737 & $5.97 \%$ \\
\hline $30 \mathrm{o} 8 \mathrm{~g} 6 \mathrm{w} 16$ & 2.304 & 2.211 & $4.21 \%$ & 2.711 & 2.947 & $8.01 \%$ \\
\hline 30o8g8w14 & 2.880 & 2.947 & $2.27 \%$ & 3.072 & 2.947 & $4.24 \%$ \\
\hline 30o12g10w8 & 3.840 & 3.684 & $4.23 \%$ & 4.608 & 4.421 & $4.23 \%$ \\
\hline
\end{tabular}

\section{Conflict of Interests}

The authors declare that there is no conflict of interests regarding the publication of this paper.

\section{Acknowledgment}

This work is supported by National Science and Technology Major Project of China (2011ZX05020-006).

\section{References}

[1] L. Zhu, N. Jin, Z. Gao et al., "Testing for nonlinearity in dynamic characteristics of vertical upward oil-gas-water three-phase bubble and slug flows," Chinese Journal of Chemical Engineering, vol. 20, no. 5, pp. 870-882, 2012.

[2] A. Wegmann, J. Melke, and P. Rudolf von Rohr, "Three phase liquid-liquid-gas flows in $5.6 \mathrm{~mm}$ and $7 \mathrm{~mm}$ inner diameter pipes," International Journal of Multiphase Flow, vol. 33, no. 5, pp. 484-497, 2007.

[3] F. Zhang and F. Dong, "A measurement method of slug flow velocity of gas-liquid two-phase flow in horizontal pipe," in Proceedings of the IEEE International Instrumentation and Measurement Technology Conference (I2MTC '10), pp. 250-254, May 2010.

[4] Y. Liu, Y. Zhang, Z. Cao, and L. Xu, "An adaptive algorithm for cross-correlation velocity measurement," in Proceedings of the IEEE Instrumentation and Control Technology (ISICT '12), pp. 96-100, 2012.

[5] C. Tan and F. Dong, "Cross correlation velocity of oil-water twophase flow by a dual-plane electrical resistance tomography system," in Proceedings of the IEEE International Instrumentation and Measurement Technology Conference (I2MTC '10), pp. $766-$ 770, May 2010.

[6] L. Kong, F. Gao, Y. Li, X. Liu, and H. J. Hai, "ICA and its application in the phase information extraction of oil-gas-water three-phase flow," in Proceedings of the 1st International Conference on Pervasive Computing, Signal Processing and Applications (PCSPA '10), pp. 549-552, September 2010.

[7] Z. Kan, X. Wang, and S. Zhang, "Study on the spatial filtering and sensitivity characteristic of inserted electrostatic sensors for the measurement of gas-solid two-phase flow parameters," Flow Measurement and Instrumentation, vol. 30, pp. 26-33, 2013.

[8] H. Seraj, M. F. Rahmat, and M. Khalid, "Measurement of velocity of solid/air two phase fluid using electrostatic sensors and cross correlation technique," Scientia Iranica, vol. 20, no. 3, pp. 786-792, 2013.

[9] F. Sapuppo, M. Bucolo, M. Intaglietta, P. C. Johnson, L. Fortuna, and P. Arena, "An improved instrument for real-time measurement of blood flow velocity in microvessels," IEEE Transactions on Instrumentation and Measurement, vol. 56, no. 6, pp. 26632671, 2007.

[10] J. Wang and D. C. Liu, "Blood flow velocity measurement: CCF based on I/Q signal," in Proceedings of the 4th International Conference on Bioinformatics and Biomedical Engineering (iCBBE '10), pp. 1-4, June 2010.

[11] G. P. Lucas and N. D. Jin, "A new kinematic wave model for interpreting cross correlation velocity measurements in vertically upward, bubbly oil-in-water flows," Measurement Science and Technology, vol. 12, no. 9, pp. 1538-1545, 2001.

[12] J. Lee, K. J. Lee, and S. K. Yoo, “Development of a new signal processing algorithm based on independent component analysis for single channel ECG data," in Proceedings of the 26th Annual International Conference of the IEEE Engineering in Medicine and Biology Society (EMBC '04), pp. 224-226, September 2004.

[13] D. S. Pham and A. M. Zoubir, "Analysis of multicomponent polynomial phase signals," IEEE Transactions on Signal Processing, vol. 55, no. 1, pp. 56-65, 2007.

[14] C. J. James and D. Lowe, "Single channel analysis of electromagnetic brain signals through ICA in a dynamical systems framework," in Proceedings of the 23rd Annual International 
Conference of the IEEE Engineering in Medicine and Biology Society, vol. 2, pp. 1974-1977, 2011.

[15] T. Huang, H. L. Wu, and C. Chen, "Fault diagnosis of rolling bearing based on the energy operator demodulation approach," in Proceedings of the 2nd International Conference on Industrial Mechatronics and Automation (ICIMA '10), vol. 2, pp. 300-303, May 2010.

[16] B. Santhanam, "Generalized energy demodulation for large frequency deviations and wideband signals," IEEE Signal Processing Letters, vol. 11, no. 3, pp. 341-344, 2004.

[17] Y. A. Hassan, J. Ortiz-Villafuerte, and W. D. Schmidl, “Threedimensional measurements of single bubble dynamics in a small diameter pipe using streroscopic particle image velocimetry," International Journal of Multiphase Flow, vol. 27, no. 5, pp. 817-842, 2001.

[18] J. R. Hopgood and P. J. W. Rayner, "Single channel nonstationary stochastic signal separation using linear time-varying filters," IEEE Transactions on Signal Processing, vol. 51, no. 7, pp. 17391752, 2003.

[19] W. Li, R. Zhang, W. Zhang, and Z. Wang, "Simulation study of two-phase gas-liquid flow capacitance-cross-correlation flow velocity measurement based on MATLAB," in Proceedings of the 2nd International Conference on Mechanic Automation and Control Engineering (MACE '11), pp. 5235-5238, July 2011.

[20] F. Dong, Y. Xu, L. Hua, and H. Wang, "Two methods for measurement of gas-liquid flows in vertical upward pipe using dual-plane ERT system," IEEE Transactions on Instrumentation and Measurement, vol. 55, no. 5, pp. 1576-1586, 2006.

[21] X.-Z. Zhang and Y. Li, "Calculation of the virtual current in an electromagnetic flow meter with one bubble using 3D model," ISA Transactions, vol. 43, no. 2, pp. 189-194, 2004.

[22] O. Cazarez, D. Montoya, A. G. Vital, and A. C. Bannwart, "Modeling of three-phase heavy oil-water-gas bubbly flow in upward vertical pipes," International Journal of Multiphase Flow, vol. 36, no. 6, pp. 439-448, 2010.

[23] F. F. Erian and L. F. Pease III, "Three-phase upward flow in a vertical pipe," International Journal of Multiphase Flow, vol. 33, no. 5, pp. 498-509, 2007.

[24] W. Zhenya, J. Ningde, Z. Yanbo, Z. Guibo, and W. Tao, "Identification of oil-gas-water three-phase flow patterns in vertical upward pipe based on multi-scale entropy," in Proceedings of the 6th International Conference on Fuzzy Systems and Knowledge Discovery (FSKD '09), vol. 5, pp. 192-196, August 2009.

[25] Y.-W. Li, L.-F. Kong, and L.-B. Liu, "Theory analysis for the virtual current distribution in an electromagnetic flowmeter with one bubble," in Proceedings of the 2nd International Conference on Information Science and Engineering (ICISE '10), vol. 2, pp. 1649-1652, December 2010.

[26] Y. Li, L. Kong, and F. Gao, "A new method for velocity measurement of oil-gas-water three-phase flow based on independent component analysis," Journal of Computational Information Systems, vol. 7, no. 4, pp. 1262-1269, 2011.

[27] S. Şişbot, "A cross-correlation technique as a system evaluation tool; application to blood flow measurement in extra-corporeal circuits," Flow Measurement and Instrumentation, vol. 16, no. 1, pp. 27-34, 2005.

[28] L.-P. Li, R.-R. Dang, D.-S. Zhao, G. Yin, and Y.-Y. Fan, "The analysis and study on electromagnetic field of conductance water fraction sensor," in Proceedings of the 3rd International Congress on Image and Signal Processing (CISP '10), vol. 9, pp. 4271-4275, October 2010.
[29] Y. Li, R. Xie, and L. Yu, "Flow pattern identification of oilgas-water three-phase flow based on NPSO-LSSVM algorithm," Journal of Theoretical and Applied Information Technology, vol. 48, no. 2, pp. 933-938, 2013. 


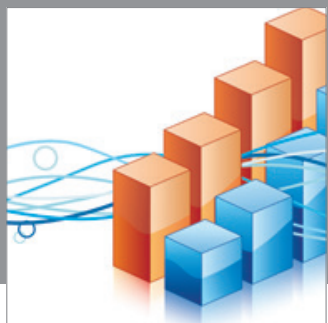

Advances in

Operations Research

mansans

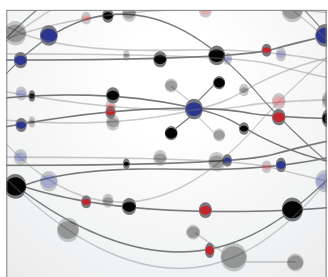

The Scientific World Journal
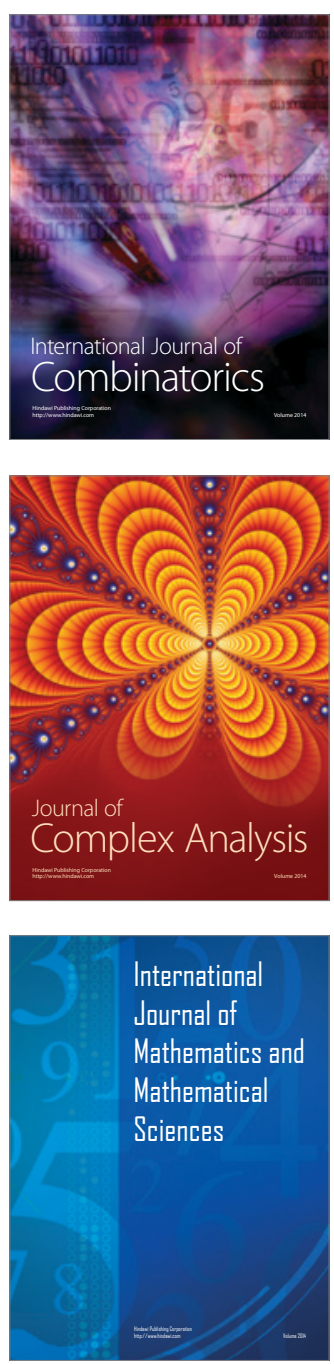
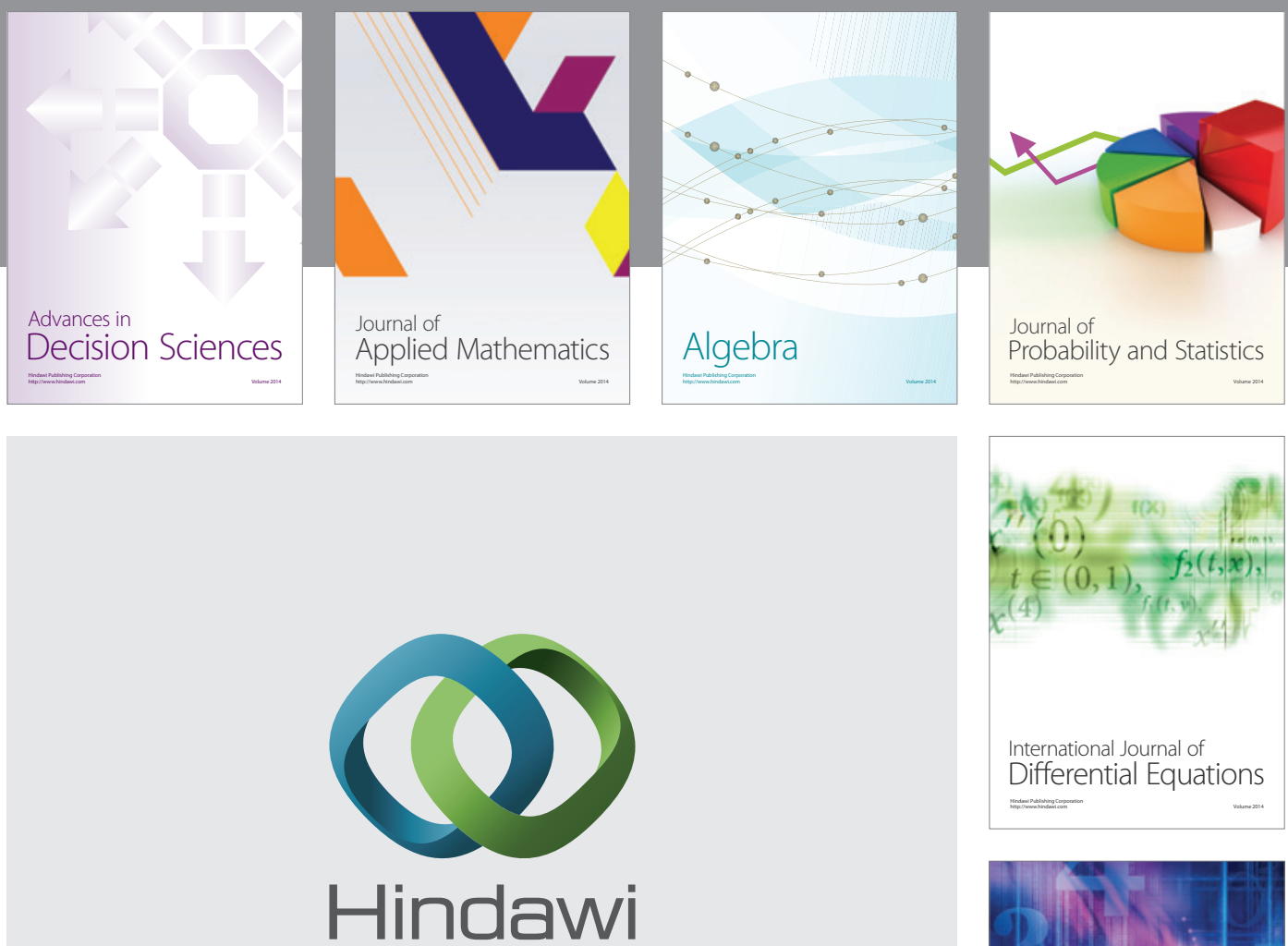

Submit your manuscripts at http://www.hindawi.com
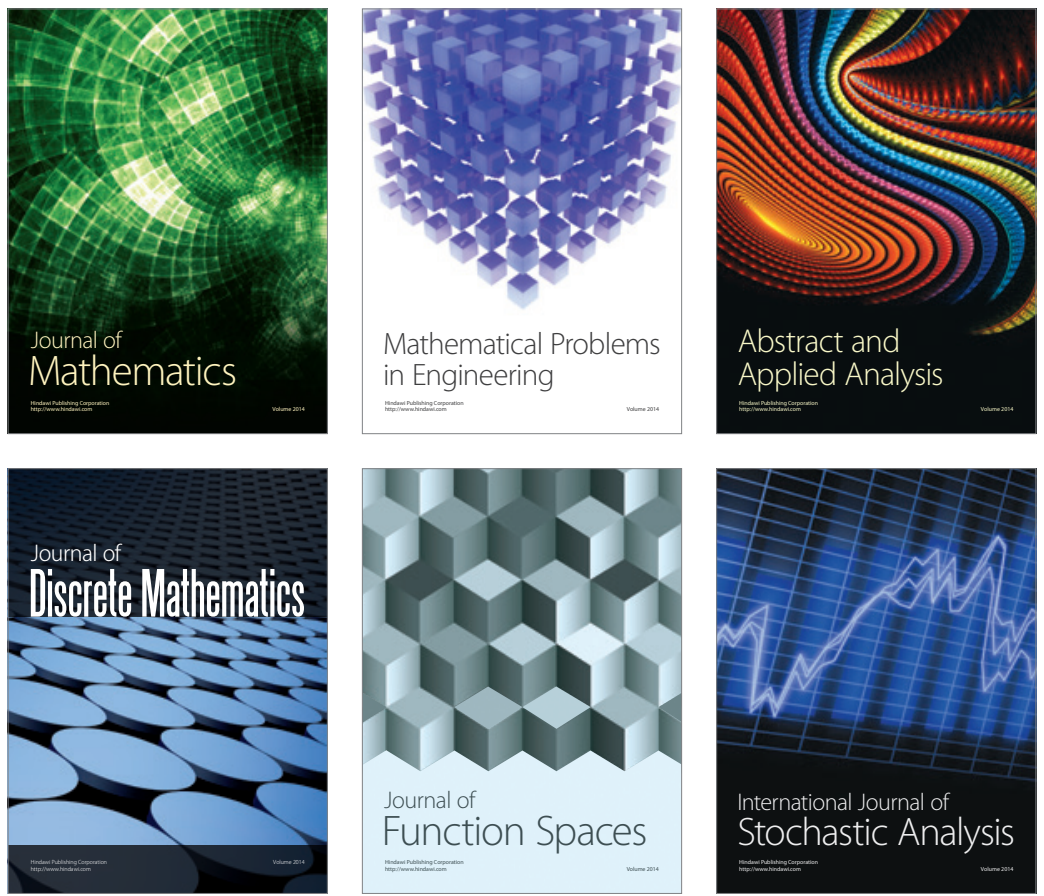

Journal of

Function Spaces

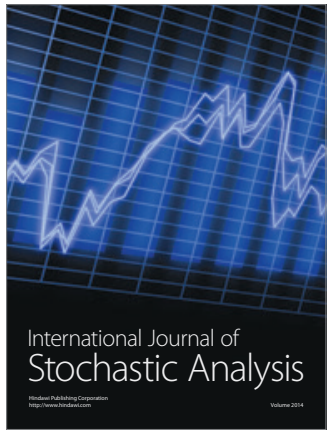

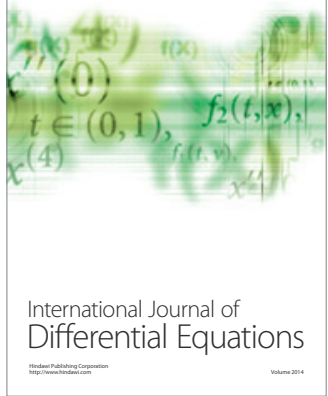
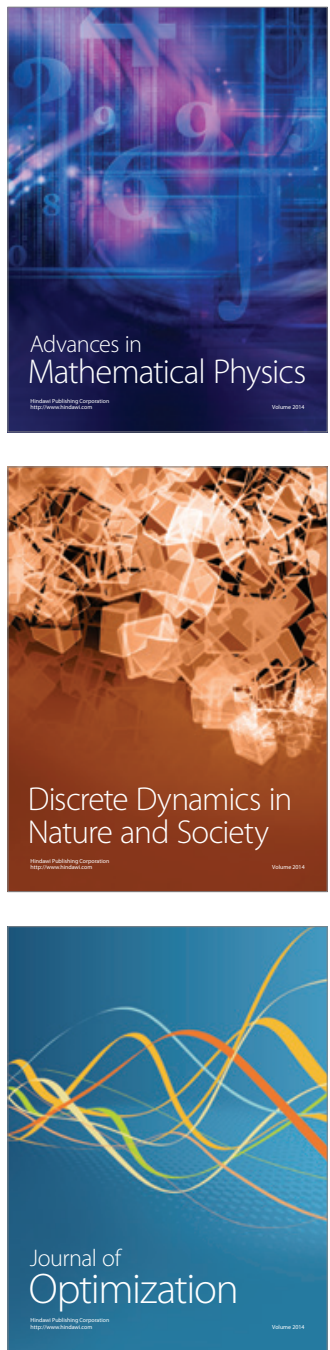